\title{
Theory-driven Hints in the Cheap Necklace Problem: A Preliminary Investigation
}

\author{
Yun Chu \\ Andrew D. Dewald \\ Edward P. Chronicle
}

\begin{abstract}
In this paper we conducted three experiments using the cheap necklace problem, which is regarded as an insight problem. The effects of two hints derived from two contemporary theoretical accounts of insight-Criterion for Satisfactory Progress theory (CSP) and Representational Change Theory (RCT) — were investigated. In Experiment 1, 78 participants made a single attempt at the problem, and significantly fewer participants given the CSP hint used an incorrect (maximizing) first move than participants given the RCT hint or control participants given no hint, Fisher's exact test for $2 \times 3$ table, $p$ $=.029$, with an approximation in $\chi^{2}$ effect size, phi $=.340$. Experiment 2 explored the performance of 60 participants in the same hint conditions over ten problem-solving trials. The number of trials to solution was significantly fewer in the CSP hint condition than in the control condition, $t(30)=2.23, p=.033, \eta^{2}=.14$; this was not so for the RCT hint condition, $t(30)=.44, p=.666, \eta^{2}=.006$. Furthermore, there were significantly fewer incorrect (maximizing) first moves in the CSP hint condition than in the other two conditions, $F(2,59)=15.31, p<.001, \eta^{2}=.35$. The CSP hint here appears to promote the exploration of the problem space, such that the correct move may be found. The lack of effect of the RCT hint suggests in preliminary fashion that representational change may not be the primary cognitive process required to solve the cheap necklace problem. However, in Experiment 3 with 110 participants, the CSP and RCT hints were combined yielding a $75 \%$ solution rate over a $34.88 \%$ solution rate in the control condition, $\chi^{2}(1)=$ $16.03, \mathrm{p}<.001, \mathrm{phi}=.402$. This result indicates that perhaps aspects from both theories are employed during the problem solving process.
\end{abstract}

Department of Psychology, University of Hawaii at Manoa.

Yun Chu, Department of Psychology, University of Hawaii at Manoa, 2430 Campus Road, Honolulu, HI 96822. Email: y.chu@hawaii.edu.

The Journal of Problem Solving • volume 1, no. 2 (Spring 2007) 


\section{The Effects of Theory-driven Hints in the Cheap Necklace Problem}

In the last ten years, there has been a rejuvenation of interest in the area of insight problem solving. Insight is often equated with the "Aha!" feeling that accompanies sudden solution attainment. There are two contemporary theoretical approaches to the cognitive mechanisms of insight: Knöblich, Ohlsson, Haider, and Rhenius's (1999; Knöblich, Ohlsson, \& Raney, 2001) Representational Change Theory (RCT) and Chronicle, Ormerod, and MacGregor's (2001; MacGregor, Ormerod, \& Chronicle, 2001; Ormerod, MacGregor, \& Chronicle, 2002; Chronicle, MacGregor, Ormerod, 2004) Criterion for Satisfactory Progress theory (CSP). RCT has its roots in the Gestalt approach, starting with Köhler's studies of problem-solving primates (1925). Ohlsson's (1992) well-known work on insight developed many of the concepts in RCT. CSP has its roots in the means-ends analysis approach and Newell and Simon's (1972) General Problem Solver model. CSP and RCT are testable and falsifiable theories that attempt to explain the cognitive processes behind insight problem solving.

\section{The Representational Change Theory}

RCT proposes that the difficulty of insight lies in the incorrect representations of the problem that hinder the ability to attain a solution (Knöblich et al., 1999). RCT predicts that perceptual restructuring plays a major role in achieving insight. RCT has focused on the importance of "chunking" when perceiving components of a problem and the phenomenon of "chunk decomposition" to find the solution. According to this theory, humans automatically organize perceived objects into groups, patterns, or chunks to facilitate their manipulation in working memory. RCT differentiates between "loose" chunks, which are somewhat difficult to break down, and "tight" chunks, which are more difficult to break down because problem solvers' previous experience with the objects led them to form "meaningful perceptual patterns." Therefore, an important factor in the insight problem's level of difficulty depends on tightness of the chunk, that is, problems containing tight chunks are more difficult to solve than problems with loose chunks. These predictions concerning chunking have been tested using equations in which Roman numerals and operators are represented by matchsticks (so-called matchstick algebra problems, Knöblich et al., 1999). In each problem, the initial equation is incorrect, and the goal state-a correct equation - may be reached by moving a single matchstick. When the matchstick that must be moved is loosely chunked, these problems are relatively easy. For example, IV = III + III becomes VI = III + III, simply by transposing the initial I. Other problems are harder: for example, $\mathrm{XI}=\mathrm{III}+\mathrm{III}$ becomes $\mathrm{VI}=\mathrm{III}+\mathrm{III}$, by sliding one of the obliques of the original $X$ over to make a $V$. Here, representational change is thought to be necessary: the solution "requires that the two tight chunks $\mathrm{X}$ and $\mathrm{V}$ are decomposed into their components" (Knöblich et al., 1999, p. 1538). Furthermore, Knöblich et al. (1999) also state that problems with narrow constraints are easier to solve than problems with wider

- volume 1, no. 2 (Spring 2007) 
constraints. Also, problems with fewer constraints are easier to solve than problems with numerous constraints. Kershaw \& Ohlsson (2004) mention other types of constraint relaxation, such as perceptual, process, or knowledge reorganization. Whether one or more of these constraint relaxation methods are used depends on the problem presented. In simple one-step matchstick problems, chunk decomposition may be the key to solution, but in multi-step problems such as the cheap necklace problem, chunk decomposition may not be enough to find the solution. Instead, a more complex set of obstacles must be surmounted and several sequential steps must be implemented to reach the goal.

\section{The Criterion for Satisfactory Progress theory}

CSP suggests that one source of difficulty with insight problems stems from the initial application of general problem solving heuristics. One such heuristic is maximizing: achieving the maximum amount of apparent progress through the problem with each move. CSP suggests that, so long as a move meets a criterion for progress (derived from the problem statement), it will be judged as satisfactory and retained. In several insight problems, however, maximizing moves are incorrect and guarantee failure to solve. For example, in the classic nine-dot problem, participants typically draw three lines around the perimeter of the dot array, canceling seven dots. This series of moves appears to make maximum progress towards the goal but cannot lead to a correct solution with the fourth line (MacGregor et al., 2001). Ormerod et al. (2002) found that solution rates were lower when initial moves that seemed to satisfy a progress criterion were available in the eight-coin problem (which requires the use of three-dimensional stacking moves for solution). Even when a hint as to the importance of three-dimensional moves was presented, criterion-satisfying moves in two dimensions predominated. However, when participants were presented with a form of the problem that interrupted maximization, the solution rate was higher than with a form of the problem that promoted maximizing.

Maximizing moves lead to apparent progress toward the solution. Participants believe they will attain solution as they continue their maximizing strategy. In reality, their maximizing is not leading to the solution, but they do not realize this until they have exhausted most of their available resources in the problem with only one or two steps remaining and they cannot reach the goal. At the time of this realization, the participants have already gone too far with their maximizing sequence of moves to backtrack and attempt a new strategy. Thus, the maximizing heuristic hinders the problem solver from experiencing criterion failure until it is too late. CSP theory talks about participants' monitoring the apparent progress towards the solution, not their actual progress. Participants will continue maximizing as long as they think they are making progress.

\section{The Cheap Necklace Problem}

Although evidence exists for both CSP and RCT accounts of insight, there have been few 
attempts to compare CSP and RCT in the same problem environment. Jones (2003) found more support for the RCT than the CSP theory. He even suggested an integration of the two theories. Ash and Wiley (2006) used problem presentations that disrupted maximization and problems that encouraged maximization and found evidence that restructuring is automatically activated. No support was found for restructuring from controlled search. The cheap necklace problem (CNP) is an attractive problem for this purpose (Silveira, 1971). The CNP requires participants to make a complete closed loop (necklace) out of 12 links of chain, with the starting point being 4 smaller, 3 -link chains. A cost constraint ( 2 cents to break a link, and 3 cents to join a link) is imposed. The correct solution involves breaking all three links of one of the 3 -link chains, and using the individual links to connect the three remaining 3-link chains together (Figure 1). A predominant first move made by naïve participants is to join two 3 -link chains together, end-to-end using 5 cents to open and close one link (Figure 1). This first move appears to maximize how much progress can be made with the first 5 cents. The problem solver determines that he/she is halfway to the solution (i.e., joining two of the four chains together) by using only one-third of the allotted money. In reality, this maximizing first move guarantees failure. Participants appear not to have the "lookahead" to realize that joining chains end-to-end would use up the 15 cents before being able to close the necklace.

CSP thus predicts that the low solution rates on the CNP are caused by the selection of maximizing first moves that appear to lead to satisfactory progress, but in fact lead to impasse if they are retained. Criterion failure is not realized until the participant has enough "lookahead" to see that 15 cents is not enough to close the necklace. Under this account, a hint that inhibits maximizing first moves should permit the participant to explore alternatives. By contrast, a plausible prediction of RCT is that difficulty of solving the CNP is due to the initial representation of the chains. The links composing the chains are not seen as individual links, but rather as parts of the chain that should not be separated (i.e., the links are hard to "dechunk" from the parent chain). Therefore, a hint to regard the links as individual objects should facilitate the dechunking of the chain into the correct representation to later use as "connectors."

In this paper, we undertake a preliminary examination of the effects of these two theory-driven hints on performance in the CNP. Experiment 1 used a single trial paperand-pencil format in an attempt to discover whether the two hints gave rise to different proportions of solutions when participants were given an extended time period in which to solve. Experiment 2 used ten shorter trials with concrete stimuli (real links and chains) in order to examine the time course of solutions. Experiment 3 combines both hints to explore the integration of the elements from the RCT and CSP theories to explain the problem solving process.

- volume 1, no. 2 (Spring 2007) 
Figure 1. The Cheap Necklace Problem (CNP). The top row shows the initial state. Following the arrows to the left and down shows the sequence of moves necessary to reach the goal state in 15c: break and separate all three links of one chain (6c) and then use the open links to join the remaining three chains into a circle (9ל). Following the arrow to the right shows the common, maximizing first move: break one link at the end of a chain (2ל) and connect to another chain using that link (3c). In addition, the correct first move and a common other first move is shown.

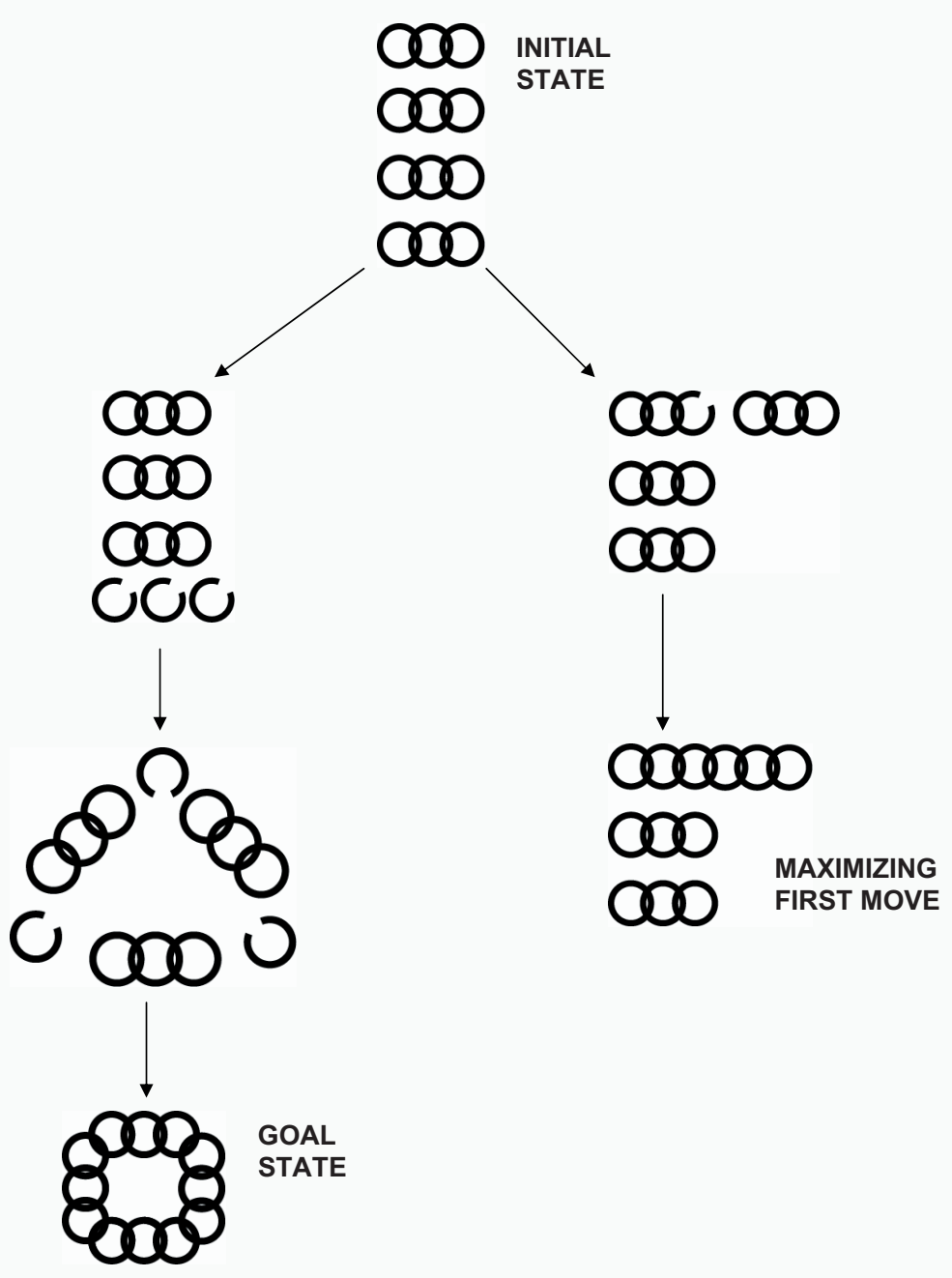

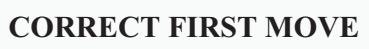

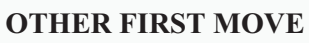

000

$\infty 0$

$\infty$

$\infty$

$\checkmark$

$\infty$

000000

め) 0

$\infty$

का

$\infty$

$\infty$

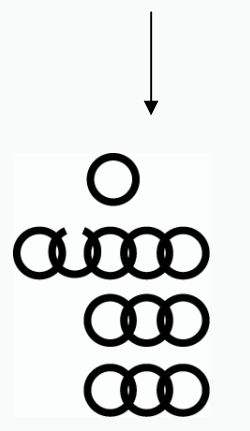




\title{
Experiment 1
}

\begin{abstract}
Method
Participants. Seventy-eight introduction to psychology students from the University of Hawaii at Manoa participated for extra class credit. No identifiers were collected and so the age range and gender ratio of the sample is unknown.

Materials. A three-page problem packet was handed out to students. The first page instructed the students to not turn the page until instructed to do so, thus all the participants could start at once and the same amount of time was given to everyone to attempt the problem. Page 2 described the CNP and gave the hint, if applicable. Page 3 had an area labeled "first move," "workspace," and "final answer." The last item in the booklet asked the students whether they had seen this or a similar problem before. Participants were instructed to sit quietly for the remainder of the time when they had finished. This statement was added to prevent a sense of competition and social demand among the participants.
\end{abstract}

Design and Procedure. The experiment was conducted in a lecture room during class time. The study used one between-subjects factor, type of hint, with three levels (control, CSP, and RCT). Students were assigned randomly to the type of hint. The dependent variables were whether the problem was solved and the type of first move (maximizing, correct, or other). Participants were given 20 minutes to work on the problem. The experimenter instructed them to feel free to use words and/or pictures to explain their answer. In the control condition, the problem was presented as seen in Figure 1. In the CSP condition, an additional sentence with the CSP hint followed the problem:

HINT: the correct first move does NOT result in a 6-link chain.

In the RCT condition, the fourth set of chains in the start state of the problem was replaced by a chain containing one red link, one blue link, and one green link. A pilot study previously demonstrated that this manipulation effectively dechunked the links. Forty-five participants were presented with six chains. Three of these had links of the same color (all blue, all green, and all red) and three had links of different colors (a combination of blue-green-red, BRG, GRB, GBR, RBG, or RGB). The participants were asked whether the picture looked more like a single chain or three links. For the same color link chains, the mean number of times they were reported as looking like a single chain was 2.04, whereas the mean for the different color link chains was 0.31 . This difference was significant, $t(44)=8.18, p<.001$.

At the end of Experiment 1, the booklets were collected, and the participants were debriefed and thanked for their participation.

- volume 1, no. 2 (Spring 2007) 


\section{Results}

There were no outliers. Data from 9 participants who stated that they had seen the problem before were excluded from analysis, resulting in 23 participants in each condition. Numbers of solutions were as follows: 10 solvers in the control condition (43.5\%), 8 solvers in the CSP condition (34.8\%), and 9 solvers in the RCT condition (39.1\%). The difference in proportion of solutions across conditions was not significant, $\chi^{2}(2)=.365, p$ $=.833, \mathrm{phi}=.073$. Participants' first moves were then scored as being maximizing (leading to a 6-link chain) or non-maximizing. There were 20 maximizing first moves in the control condition (87.0\%), 14 in the CSP condition (60.9\%), and 21 in the RCT condition (91.3\%). As three cells had expected frequencies of less than 4, these data were analyzed by a Fisher's exact test for a $2 \times 3$ table (Lowry, 2006). The difference among conditions was significant, $p=.029$, with an approximation in $\chi^{2}$ effect size, phi $=.340$. Follow-up Fisher's exact tests showed that the proportion of maximizing first moves was lower in the CSP condition than in the control condition $(p=.045)$, and not significantly different in the RCT and control conditions ( $p=.50)$.

\section{Discussion}

Experiment 1 found no significant differences between control, CSP and RCT hint in the proportion of solutions to the CNP. This result was unexpected and seems to imply that neither of the hints was effective. It is possible, however, that the 20-minute period given for attempting the problem was too long, such that differences between conditions were obscured. The format of the experiment did not permit the assessment of time to solution.

There was a significant difference across conditions in the proportion of first moves that were of the maximizing type (that is, joined two 3-link chains end to end to form a 6 -link chain). The CSP hint significantly reduced the proportion of such moves. Interestingly, however, it did not appear to increase the number of correct first moves: there were only three such moves in the CSP condition (compared with one in the control and two in the RCT conditions). We return to this issue in the General Discussion.

In order to assess time to solution, Experiment 2 used a method in which participants undertook ten separate trials of two minutes each, with the problem being reset to the initial state at the start of each trial. This permitted the examination of how quickly solutions emerged, and the types of first moves over trials.

\section{Experiment 2}

\section{Method}

Participants. Sixty volunteers from the University of Hawaii at Manoa were recruited. No identifiers were collected, as in Experiment 1. All participants were naïve to the CNP and 
fluent in English. Participants were compensated $\$ 2$ for every 15 minutes for their time and inconvenience.

Apparatus. The CNP comprised four sets of metal chains with three links, each link being an oval-shaped 1/8" ( $3 \mathrm{~mm})$ "quick repair" link. These links may be opened and closed by unscrewing and screwing up a threaded component on one side of the link. For the $\mathrm{RCT}$ hint condition, an additional three links were spray-painted red, blue, and green. Participants had available a sheet with fifteen pennies drawn on it in order to keep track of how much money was remaining for the problem. Each trial was timed with a handheld stopwatch.

Design and Procedure. The experiment was conducted with individual participants in a cognitive psychology laboratory at the University of Hawaii at Manoa. The study used one between-subjects factor, type of hint, with three levels (CSP, RCT, and control). Participants were randomly assigned to one of the three conditions. The dependent variables were whether the problem was solved, the number of trials to solution, and the type of first move. Participants were seated next to the experimenter, who ensured that they understood the instructions, answered any questions, and kept track of the number of trials, moves, and money spent on the moves. The four metal chains were placed on the desk in front of the participant. A square area of about 18 inches was taped off on the desk to denote workspace with the chains. The experimenter showed the participants how to open and close the links.

The same problem description from Experiment 1 was used. In the control condition, participants were given the instructions both on a sheet and orally by the experimenter. In the CSP condition, an additional sheet of paper with the same "do not maximize" hint as in Experiment 1 was given to the participant. The hint was also orally explained by the experimenter. In the RCT condition, the fourth set of chains presented to the participant contained one red link, one blue link, and one green link. The rest of the problem was the same as the control condition.

The participants were informed that they had 10 trials and each trial consisted of any number of moves made by the participant until they either exceeded the 15 cents or 2 minutes ran out. After the instructions were given and the participants' questions were answered, the experimenter said "begin" and the time started. The first move for each trial was recorded by the experimenter. Each time the participant opened a link, the experimenter crossed out 2 pennies on the penny sheet in front of the participant. Each time the participant closed a link, 3 pennies were crossed out. The experiment ended when the participant either solved the problem 3 times in a row or the 10 trials were finished. At the end of the experiment, participants were debriefed, compensated, and thanked for their participation.

- volume 1, no. 2 (Spring 2007) 


\section{Results}

There were no outliers. In both of the control and CSP conditions, 16 of 20 participants solved the problem; 15 of 20 solved in the RCT condition. The proportion of solvers across conditions was not significant (Fisher's exact test for $2 \times 3$ table, $p=1.0$ ).

The mean numbers of trials to solution were as follows: control 5.63 (2.87), CSP 3.38 (2.66), RCT 4.93 (2.81). Trials to solution data were analyzed with a one-way analysis of variance to test the generic null hypothesis and two planned comparisons using $t$-tests (control versus CSP, and control versus RCT) to test for specific contrasts. There was a marginally non-significant overall effect of hint condition on number of trials to solution, $F(2,46)=2.84, p=.069, \eta^{2}=.11$. The number of trials to solution was significantly lower in the CSP condition than in the control condition, $t(30)=2.23, p=.033, \eta^{2}=.14$, but not significantly lower in the RCT condition than in the control condition, $t(30)=.44$, $p=.666, \eta^{2}=.006$.

The proportion of maximizing first moves (number of trials with a maximizing first move/total number of trials undertaken) was calculated for each participant, and the resultant proportions compared across conditions with one-way analysis of variance and planned comparisons as above. Mean proportions were as follows: control $0.56(0.20)$, CSP 0.16 (0.28), RCT 0.59 (0.29). The difference in mean proportion across hint conditions was significant, $F(2,59)=15.31, p<.001, \eta^{2}=.35$. The proportion of maximizing first moves was lower in the CSP condition than the control condition, $t(39)=4.66, p<.001$, $\eta^{2}=.36$, but not significantly lower in the RCT condition than in the control condition, $t(39)=-0.68, p=0.43, \eta^{2}=.016$. Figure 2 shows trial-by-trial proportions of participants making maximizing (panel A) correct (panel B) and other (panel C) first moves in each condition. A maximizing first move involved connecting two chains end-to-end forming a 6-link chain. A correct first move involved disconnecting one link and using that link as a "connector" by putting the link through two other chains, forming a 7-link chain. Other first moves included any combination that was not maximizing or correct. For example, the most common other first move is disconnecting the middle link from a chain and removing one of the intact links on either side, leaving a 2-link chain with one of the links open. Most participants starting this way often connected the 2-link chain to another 3-link chain, making a 5 -link chain.

For example, a typical control participant (\#3- did not solve) would try the following sequence of first moves across 10 trials: T1- maximizing; T2- opened one of the end links on one chain and inserted it through the middle link of another chain making a 6-link Tshaped form; T3- opened the middle link of one chain and connected it to the end link on the right of another chain making a T-shape as in T2, but in "reverse" form; T4- same as T3, but connecting the opened middle link to the end link on the left of another chain. Trials 5-10 were all maximizing first moves. Participants tried linking pieces from different sides, but never attempted to disconnect one of the opened links. Participants' conservative 
Panel A

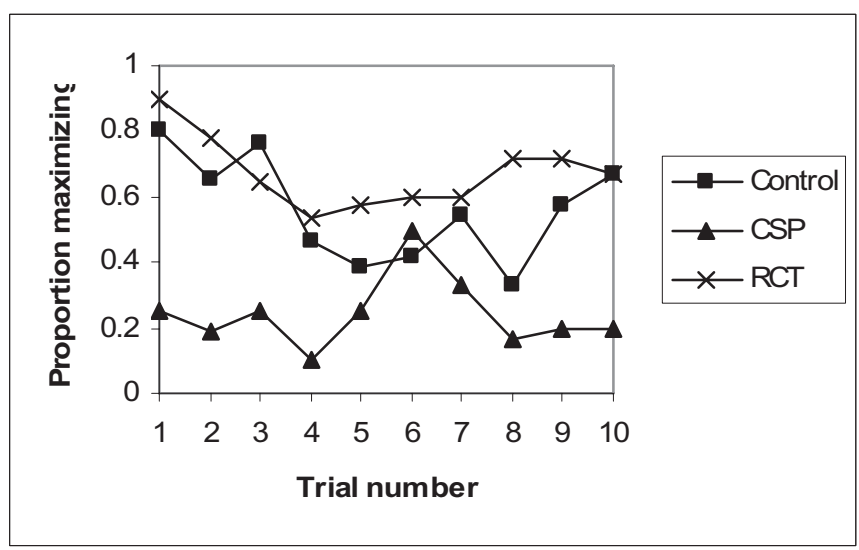

Panel B

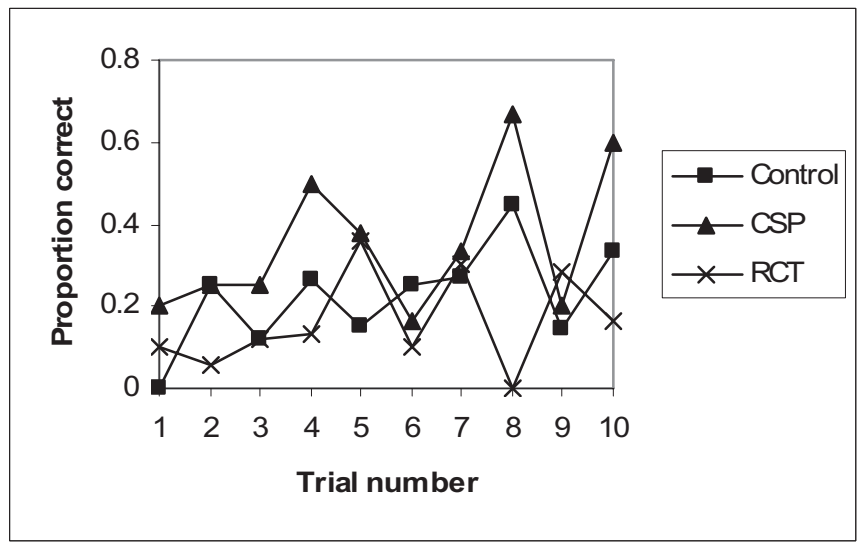

Panel C

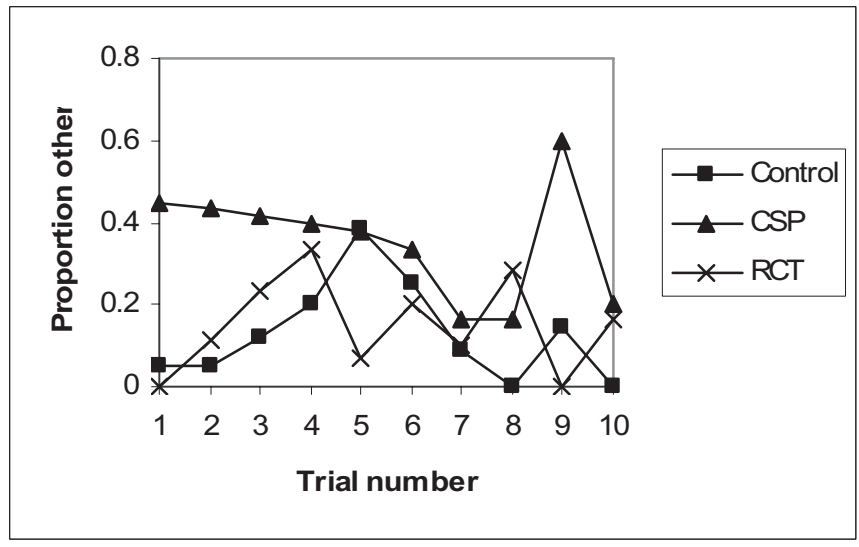

Figure 2. Proportions of maximizing first moves (Panel A), correct first moves (Panel B) and other first moves (Panel $C$ ) at the beginning of each trial across the ten trials of Experiment 2 , separated by hint condition.

- volume 1, no. 2 (Spring 2007) 
approach always resulted in a 6-link chain after the maximizing first move. An example of a participant in the RCT condition (\#48- solved on T7) would be as follows: T1-T3 were maximizing first moves; T4- the most common "other" first move making a 5-link chain (Figure 1); T5-T6 repeated T4 with small variations in moves following the "other" first move; T7- the correct first move resulting in a 7-link chain (Figure 1) followed by opening of another link on the first chain and eventually leading to the solution. An example of a participant in the RCT condition (\#34- solved on T3) had the following sequence of first moves: T1- the most common "other" first move making a 5-link chain; T2- opened the end link of a chain, opened the end link of another chain, opened the end link of a third chain and connected it to the end of the last chain making a 6-link chain; T3-opened all 3 links on one chain and arrived at the solution. The CSP hint alerted participants of criterion failure (maximizing will not work) at the beginning of the problem, allowing them to try other moves, eventually leading to solution, while in the control and RCT hint conditions, the participants attempted to maximize at least for some of their trials.

\section{Discussion}

As in Experiment 1, the proportion of participants solving the problem was not significantly different across hint conditions. We hypothesize that only recording solution rates is not a sensitive enough method to ascertain the effects of hints on problem performance. However, participants solved the problem in fewer trials in the CSP condition than the control condition; this was not so for the RCT condition. Consistent with Experiment 1, the proportion of maximizing first moves was lowest in the CSP condition. It is notable from Figure 2, panel A that participants in the CSP condition avoid the maximizing first move from trial 1: the CSP hint is effective in this regard. However, the proportion of correct first moves in trials 1-3 is not substantially higher in the CSP condition than the other two conditions (Figure 2, panel B). It appears that the CSP hint allows participants to explore other moves (Figure 2, panel C) but does not automatically lead to selection of a correct first move.

The sudden upshot in maximizing first moves (panel A) in the CSP condition is attributed to participants' just "having to try" the maximizing move that they have wanted to do since the beginning but have been warned against. By observing the participants one-on-one, we determined that they almost seem not to believe the experimenter that the CSP hint helps solution. Participants in the CSP condition start by avoiding maximization, but since other moves have not led to the solution yet (trials 1-5), they make a last effort to try everything, including joining two chains end-to-end. Once satisfied that in fact this 6-link chain first move does not work, they continue exploring other first moves as before.

Through verbal protocol, we found that participants did not attribute their success or lack thereof to the hints. Participants' first reaction to the CSP hint was confusion. They 
could not see how not making a 6-link chain could lead to the solution. Participants did not have much of a reaction to the colored links in the RCT condition. Most thought they did not mean anything, and some even thought the colors were distractors. Solvers were asked whether the hints helped in the CSP and RCT conditions. Some participants in the CSP said yes. They stated that even though they did not quite understand the hint at the beginning, they eventually found the right moves. Very rarely did participants in the RCT condition say that the colored links helped. During debriefing when the RCT hint was explained to help by dechunking, participants could see the reason for the hint but still stated that it did not help their own problem solving experience. However, participants may not have adequate metacognition into their problem solving process.

A final issue is why participants in the CSP condition would attempt any maximizing moves at all if they were explicitly directed not to do so. Our best explanation from having watched hundreds of participants in these experiments and several others involving a "do not maximize" hint on insight problems is that participants' desire to maximize is extremely strong. Their desire to maximize even overrides directions from the experimenter. Since most participants cannot find a more "productive" move than joining two chains end-to-end, they cannot imagine the maximizing move to be incorrect. Participants will persist with the maximizing first move, thinking that it must be correct and the "trick" to solving the problem must lie in changing later moves. Through verbal protocol, we find that participants immediately perform the maximizing first move and only then do they pause to determine how else to join the rest of the chains. Participants attempt to find a way to "save" money at the end of the problem to accomplish the closed necklace for 15 cents. Although the CSP hint is clearly stated and emphasized to the participants, the experimenter cannot force the hand of the participants as they are maximizing. Some participants open one link and immediately connect it to another chain. The experimenter notes this move and reminds the participant of the CSP hint for future reference. The experimenter cannot ignore this first move and record only what happens after the participant is forced to try another move. It is worth noting that maximization is such a strong force that some participants will even disregard the experimenter's instructions.

Although effects of hint condition on total number of solutions did not emerge in either condition, Experiment 2 demonstrated that the CSP hint allowed solutions to be discovered more quickly: on average, about two trials sooner than in the control condition. The CSP hint effectively steers participants away from a maximizing first move. It might be objected that the CSP hint "gives the game away": if this were the case, it would be expected that first moves in this condition would be overwhelmingly correct. This was not the case (Figure 2). Rather, it seems that the CSP hint allows participants to avoid a compelling (but incorrect) first move, and to explore the problem space. This exploration leads to solution sooner than in the other two conditions. Not all the participants

- volume 1, no. 2 (Spring 2007) 
in the CSP condition solved because the hint inhibits maximization for most, but does not guarantee solution, as the CNP is a multi-step problem. Some participants were able to achieve the correct first move, only to become confused in later steps to necklace completion. Therefore, Experiment 3 was devised to investigate the effects of combining both hints on the solution process. Perhaps different aspects from the two hints are necessary to attain solution.

\section{Experiment 3}

Experiment 3 was undertaken to explore whether combining the RCT and the CSP hints would increase the solution rate. Jones (2003) suggests that CSP theory explains how people seek insight with improved "lookahead" and by monitoring progress, while RCT explain how constraint relaxation achieves solution. In sum, both theories help explain different aspects of the complex problem solving process.

\section{Method}

Participants. One hundred ten University of Hawaii at Manoa undergraduate students in a psychology class participated for extra credit.

Design and Procedure. A between-subjects design with 51 participants in the control condition and 59 participants in the combined hint condition (CSP + RCT hints) were given one sheet of paper describing the CNP. The combined hint condition differed from the control condition with the last chain in the initial state made up of a red, a blue, and a green link. In addition, an explicit sentence noted that the colored links could help solve the problem. The CSP hint is the same as in the previous 2 experiments. The participants were given 15 minutes to solve the problem.

\section{Results}

There were no outliers. Eleven participants were excluded from the data analyses because they had previously seen the problem. Forty-three participants were in the control condition. Fifty-six participants were in the combined hints condition. In the control condition 15 participants solved (34.88\%). In the combined hints condition, 42 participants solved (75.00\%). There was a significant different in solution rates between the conditions, $\chi^{2}(1)$ $=16.03, p<.001$, phi $=.402$.

\section{General Discussion}

Three experiments were undertaken in which the effectiveness of hints derived from predictions of CSP and RCT theories were compared in a single problem, the CNP. Pilot data demonstrated that the RCT hint effectively perceptually dechunks the three links of one chain in the start state of the problem. This might seem a very obvious hint; however, 
it does not appear to be sufficient to overcome the maximizing heuristic; participants in the RCT condition were similar to those in the control condition with regard to the proportion of maximizing first moves. The lack of effect of the RCT hint is intriguing in Experiments 1 and 2 and appears similar to previous work demonstrating no effect of a salient perceptual hint in the 9-dot problem (Chronicle et al., 2001).

We acknowledge, however, that the presentation of CSP and RCT hints differed in other dimensions: one was oral, the other visual; one gave an explicit procedural instruction, the other did not. It will be important to investigate the influence of these dimensions in future work. Nonetheless, the results of both experiments underscore the importance of the maximizing heuristic (MacGregor et al., 2001; Ormerod et al., 2002; Chronicle et al., 2004) for understanding performance in insight problems. Examining control conditions alone, in Experiment 1, 87\% of first moves were maximizing. In the first trial of Experiment 2, $80 \%$ were maximizing; furthermore, the proportion of participants employing a maximizing first move never dropped below 0.33 across ten trials. We argue that the maximization heuristic is engaged, and remains engaged, because it results in moves that meet a criterion for satisfactory progress in problem environments. In the CNP, the goal state is a closed 12-link necklace. The maximizing first move of joining chains end-to-end to give a 6-link chain appears to make good progress: the 6-link chain is halfway to the goal length for an expenditure of only a third of the money available. In this manner, a state of impasse is reached, and only when maximization is prevented may the impasse be broken and other moves explored. At this point, perhaps the RCT hint aids in solution by relaxing constraints and allowing the participants to open the other two links to use as connectors. Because CNP is a multi-step problem, several elements make the solution hard to attain. Neither the CSP nor the RCT hints alone can increase the solution rate. In order to fully explain the mechanisms behind a complex insight problem, Jones (2003) might have hit on an important point regarding the integration of both theories. However, this is a new perspective in the insight problem solving area, and further experiments will be needed to investigate these conclusions.

\section{Author Note}

The authors would like to thank Trina Kershaw and an anonymous reviewer for insightful revision suggestions that made this article more comprehensive.

\section{References}

Ash, I. K. \& Wiley, J. (2006). The nature of restructuring in insight: An individual differences approach. Psychonomic Bulletin \& Review, 13(1), 66-73.

Chronicle, E. P., MacGregor, J. N., \& Ormerod, T. C. (2004). What makes an insight problem? 
The role of heuristics, goal conception, and solution recoding in knowledge-lean problems. Journal of Experimental Psychology: Learning, Memory, and Cognition, 30, 14-27.

Chronicle, E. P., Ormerod, T. C., MacGregor, J. N., Dewald, A. D., \& Chu, Y. The locus of insight: Problem, process, or outcome? Memory \& Cognition. (submitted).

Chronicle, E. P., Ormerod, T. C., \& MacGregor, J. N. (2001). When insight just won't come: The failure of visual cues in the nine-dot problem. The Quarterly Journal of Experimental Psychology A, 54, 903-919.

Jones, G. (2003). Testing two cognitive theories of insight. Journal of Experimental Psychology: Learning, Memory, and Cognition, 29, 1017-1027.

Kershaw, T. C. \& Ohlsson, S. (2004). Multiple causes if difficulty in insight: The case of the nine-dot problem. Journal of Experimental Psychology: Learning, Memory, and Cognition, 30(1), 3-13.

Knöblich, G., Ohlsson, S., Haider, H., \& Rhenius, D. (1999). Constraint relaxation and chunk decomposition in insight problem solving. Journal of Experimental Psychology: Learning, Memory, and Cognition, 25, 1534-1555.

Knöblich, G., Ohlsson, S., \& Raney, G. E. (2001). An eye movement study of insight problem solving. Memory \& Cognition, 29, 1000-1009.

Köhler, W. (1925). The mentality of apes. New York: Harcourt \& Brace.

Lowry, R. (2006). VassarStats website for statistical computation [web-based software and manual]. Retrieved from http://faculty.vassar.edu/lowry/VassarStats.html.

MacGregor, J. N., Ormerod, T. C., \& Chronicle, E. P. (2001). Information processing and insight: A process model of performance on the nine-dot and related problems. Journal of Experimental Psychology: Learning, Memory, and Cognition, 27, 176-201.

Newell, A. \& Simon, H. A. (1972). Human problem solving. Englewood Cliffs, NJ: PrenticeHall.

Ohlsson, S. (1992). Information-processing explanations of insight and related phenomena. In M. Keane \& K. Gilhooley (Eds.), Advances in the psychology of thinking (pp. 1-44). London: Harvester-Wheatsheaf.

Ormerod, T. C., MacGregor, J. N., \& Chronicle, E. P. (2002). Dynamics and constraints in insight problem solving. Journal of Experimental Psychology: Learning, Memory, and Cognition, 28, 791-799.

Silveira, J. M. (1971). Incubation: The effect of interruption timing and length on problem solution and quality of problem processing. Unpublished doctoral dissertation, University of Oregon.

Paper submitted on January 9, 2007.

The final version accepted on March 26, 2007. 